\title{
Transformation of hashimoto thyroiditis to Graves' disease
}

\author{
Stella Pak, Damian Valencia and Adam Fershko* \\ Department of Medicine, Kettering Medical Center, Kettering, 3535 Southern Blvd, Kettering, OH 45429, USA
}

\begin{abstract}
Hashimoto's thyroiditis and Graves' disease are thyroid-specific autoimmune disorders with distinct pathological mechanism. Clinical presentation and histopathological features also vary greatly in these disorders. Sequential conversation from Hashimoto's thyroiditis (HT) to Graves' disease (GD) has been rarely reported throughout the world. Herein, we report a woman with history of HT, who then developed GD requiring total thyroidectomy. By reporting this case, we hope to raise awareness about this phenomenon among clinicians and illustrate the potential mechanisms behind such change. Improving recognition and identification of this potential conversation between HT and GD would allow clinicians to expeditiously implement appropriate treatment, increasing the quality of care.
\end{abstract}

\section{Introduction}

Hashimoto thyroiditis (HT) is a chronic inflammatory disorder of thyroid glands. HT causes hypothyroidism and affects approximately $0.8 \%$ of the population. Anti-thyroperoxidase antibodies and antithyroglobulin antibodies are found in $95 \%$ and $60-80 \%$ of patients with HT, respectively [1]. These autoantibodies cause progressive destruction of thyroid follicles, eventually resulting in atrophy and fibrosis of thyroid glands. CD4 (+) T-Helper-1 (TH-1) cells play a major role in the pathogenesis of HT [2].

Contrarily, Graves' disease (GD), an autoimmune process which leads to hyperthyroidism, is characterized by the presence of antithyrotropin receptor antibodies and clinically by exophthalmos and pretibial myxedema. GD affects about $0.5 \%$ of the population and can possibly cause thyrotoxicosis [3,4]. In GD Autoantibodies overstimulates thyroid-stimulating hormone (TSH) receptors, resulting in hypertrophy of thyroid glands. Unlike HT, TH-2 cells play a key role in disease process of GD [2]. Because different autoantibodies and TH cells are involved, the conversion between HT and GD is rare; but, yet a sequential conversion of HT to GD is even more rare [4]. Herein, we report a woman with history of HT, who then developed GD that required a total thyroidectomy.

\section{Case presentation}

The patient is a 41-year-old female with a past medical history of Hashimoto thyroiditis who presented with new-onset palpitation, unintentional weight loss (approximately $10 \mathrm{lbs}$.), hair loss, fatigue, constipation and tremors over the past 5 months. On presentation, the patient vitals were: blood pressure $148 / 82 \mathrm{mmHg}$, heart rate 72 / min, respiratory rate $16 / \mathrm{min}$, temperature $37^{\circ} \mathrm{C}$ and oxygen saturation of $98 \%$. Initial laboratory results revealed TSH $0.02 \mathrm{ng} / \mathrm{dL}, \mathrm{T}_{4}$ of 1.71 $\mathrm{ng} / \mathrm{dL}$, and $\mathrm{T}_{3} 3.2 \mathrm{ng} / \mathrm{dL}$ which were significantly different from her baseline (TSH $3.73 \mathrm{ng} / \mathrm{dL}, \mathrm{T}_{4}$ of $1.08 \mathrm{ng} / \mathrm{dL}$, and $\mathrm{T}_{3} 2.70 \mathrm{ng} / \mathrm{dL}$ ). After initial evaluation, the patient's home levothyroxine (112 $\mathrm{mg}$ daily) was discontinued and she was started on propranolol (10 mg daily). From initial onset of hypothyroidism, she continued to be sero-negative for thyroperoxidase antibody. On the other hand, serology test was positive for thyroid stimulating antibodies, at $3.93 \mathrm{IU} / \mathrm{L}$, diagnostic of Graves' disease. Her thyroid stimulating immunoglobin level was also 164 units.
The ultrasonogram of her thyroid revealed a bilateral multinodular goiter and heterogeneous echotexture (Figure 1). On her radioactive studies, she had heterogeneous uptake. Radioiodine ablation was not possible due to her iodine allergy and the heterogeneous uptake within the gland. Thus, our patient underwent a total thyroidectomy. Pathological examination of removed thyroids revealed abundant thyroglobulin, consistent with the diagnosis of hyperthyroidism (Figure 2). She had uneventful postoperative recovery, but developed postoperative hypothyroidism. Her laboratory results were TSH 2.73 $\mathrm{ng} / \mathrm{dL}, \mathrm{T}_{4}$ of $0.74 \mathrm{ng} / \mathrm{dL}$, and $\mathrm{T}_{3} 2.70 \mathrm{ng} / \mathrm{dL}$. As a result, the patient was started on levothyroxine (112 mg daily) and now remains symptom free.

\section{Discussion}

When the patient with HT presents with symptoms of hyperthyroidism, the most likely cause is over-replacement of thyroid hormone. However, as highlighted in the present case, it is crucial to include GD in the differential diagnosis, given the common autoimmune origin of both disorders. As GD can cause irreversible complications such as exophthalmos, timely diagnosis and treatment is important to improve and maintain the quality of lives of patients [3].

The pathophysiology underlying the conversion of HT to GD remains largely unknown. There are three plausible hypotheses for this rare transformation. First hypothesis postulates that the development of various autoantibodies can give rise to intricate interaction between multiple types of antibodies and thyroid glands, leading to the conversion. Second hypothesis is that the thyroid dysfunction presented as hypothyroidism from severe autoimmune-induced tissue injury, but then later thyroid glands gradually recover from the injury and become able to response to thyroid stimulating antibodies, leading to hyperthyroidism [5]. Third possible hypothesis is that age-related

Correspondence to: Stella Pak, MD, Department of Medicine, Kettering Medical Center, Kettering, 3535 Southern Blvd, Kettering, OH 45429, USA, Tel. (937) 395-8997; Fax: (937) 395-8399; E-mail: Stella.Pak@ketteringhealth.org

Key words: hashimoto thyroiditis, graves' disease, thyroidectomy

Received: October 08, 2017; Accepted: October 23, 2017; Published: October 26, 2017 


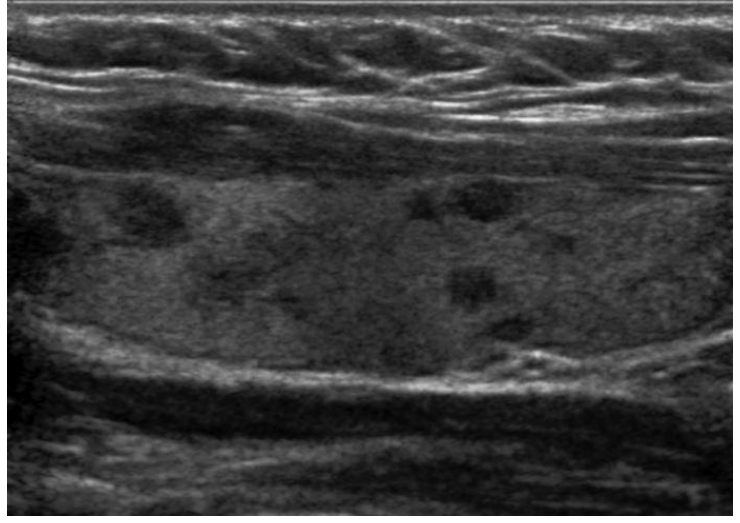

Figure 1. Ultrasonogram of left thyroid demonstrates multiple nodules and heterogeneous echotexture.

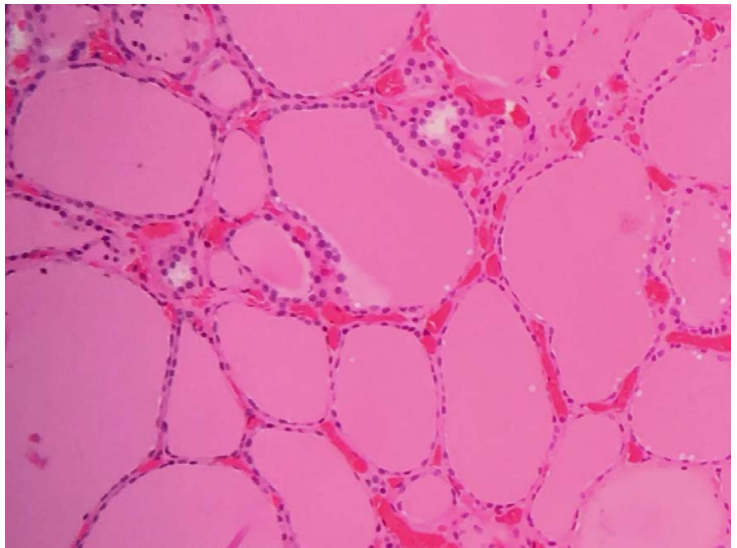

Figure 2. Thyroid tissue specimen at $10 \mathrm{X}$ magnification. Note the abundant amount of thyroglobulin.

change in immunomodulatory state can drive the transformation between these autoimmune disorders. This is highly unlikely in our patient as she is not in elderly age group. Associated factors with the conversion process are pregnancy and the use of immunomodulatory drugs, such as Alemtuzumab [6].
Hypothyroidism from HT was traditionally believed to result from the gradual destruction of thyroid follicles by autoantibodies [7]. Thus, the sequential conversation from HT to GD challenges the current view in pathophysiology of HT. In the patients who had a shift from HT to GD, the autoantibodies could have merely blocking the TSH receptors rather than infiltrating and destructing the thyroid glands. Accumulation and analysis of cases of the rare transformation would enable unraveling of its pathophysiology. The reason for lower incidence of conversion from HT to GD than the other way around is not known [4]. The symptoms of HT tend to be mild and, thus, more likely to be overlooked than that of GD. Based on this, the transformation from HT to GD may not be as rare as inferred by literature, but, rather, missed.

In summary, we described a case of a sequential transformation from HT to GD. By reporting this case, we hope to raise the awareness about this rare phenomenon among clinicians and illustrate the potential mechanisms behind such change. Improvement of recognition and identification of this conversation process between HT and GD would allow clinicians to expeditiously implement appropriate treatment, thus increasing the quality of care.

\section{References}

1. Caturegli P, De Remigis A, Rose NR (2014) Hashimoto thyroiditis: clinical and diagnostic criteria. Autoimmun Rev 13: 391-397. [Crossref]

2. Marique L, Van Regemorter V, Gérard AC, Craps J, Senou M, et al. (2014) The expression of dual oxidase, thyroid peroxidase, and caveolin-1 differs according to the type of immune response (TH1/TH2) involved in thyroid autoimmune disorders. J Clin Endocrinol Metab 99: 1722-1732. [Crossref]

3. Smith TJ, Hegedüs L (2016) Graves' Disease. N Engl J Med 375: 1552-1565. [Crossref]

4. Holt K (2010) Graves' disease: clinical pathophysiology, presentation and treatment options. J Pract Nurs 60: 13-18. [Crossref]

5. Furqan S, Haque NU, Islam N (2014) Conversion of autoimmune hypothyroidism to hyperthyroidism. BMC Res Notes 7: 489. [Crossref]

6. Kamath C, Young S, Kabelis K, Sanders J, Adlan MA, Furmaniak J, et al. (2012) Thyrotrophin receptor antibody characteristics in a woman with long-standing Hashimoto's who developed Graves' disease and pretibial myxoedema. Clin Endocrinol (Oxf) 77: 465-470. [Crossref]

7. Chung YH, Ou HY, Wu TJ (2004) Development of hyperthyroidism following primary hypothyroidism: a case report. Kaohsiung J Med Sci 20: 188-191. [Crossref]

Copyright: (C2017 Pak S. This is an open-access article distributed under the terms of the Creative Commons Attribution License, which permits unrestricted use, distribution, and reproduction in any medium, provided the original author and source are credited. 